\title{
Comprehensive portrait of recurrent glioblastoma multiforme in molecular and clinical characteristics
}

\author{
Rui $\mathrm{Li}^{1}$, Xincheng Chen ${ }^{1}$, Yongping You ${ }^{1}$, Xiefeng Wang ${ }^{1}$, Yanwei Liu ${ }^{2}$, Qi Hu${ }^{1}$, \\ Wei Yan ${ }^{1}$ \\ ${ }^{1}$ Department of Neurosurgery, The First Affiliated Hospital of Nanjing Medical University, Nanjing, China \\ ${ }^{2}$ Beijing Neurosurgical Institute, Capital Medical University, Beijing, China \\ Correspondence to: \\ Wei Yan, e-mail: neuro_yw@njmu.edu.cn \\ Keywords: recurrent glioblastoma, primary glioblastoma, molecular characteristics, clinical features \\ Received: March 25, $2015 \quad$ Accepted: August 25, $2015 \quad$ Published: September 04, 2015
}

\section{ABSTRACT}

Glioblastoma multiforme is the most common primary malignant brain tumor in adults. In addition to poor response to treatment, a high recurrence rate contributes to the poor prognosis. The purpose of this study was to investigate the genetical and clinical characteristics of recurrent glioblastoma. We used whole transcriptome sequencing data to examine the distribution of molecular subtypes and gene signatures in 22 recurrent glioblastoma taken from the Chinese population, and further analyzed biological progression of the tumors, when compared with primary glioblastoma. The proportion of the classical subtype in recurrent ones $(22 \%)$ was lower than that in primary glioblastoma $(36 \%)$. The frequency of IDH1 mutations in recurrent glioblastomas was nearly twice that in primary glioblastomas. TP53 mutations were fewer in proneural recurrent glioblastomas $(20 \%)$ but frequent in classical recurrent glioblastomas $(80 \%)$. The most common sites of recurrent glioblastomas were the temporal lobe $(41 \%)$. In patients diagnosed with recurrent glioblastoma multiforme, 64\% were younger than 50 years. Gene set enrichment analysis revealed that chromatin fracture, repair, and remodeling genes were enriched in recurrent glioblastoma. Our results highlight the differences in clinical features, molecular subtypes and gene alterations between primary and recurrent glioblastoma and may be helpful for targeted therapy for recurrent glioblastoma.

\section{INTRODUCTION}

Glioblastoma multiforme (GBM) is the most common and malignant primary brain tumor in adults. Despite advancing treatments including resection, radiotherapy and chemotherapy, most patients survive less than one year [1-4]. Numerous patient-specific factors result in a poor response to treatment, including the tumor acquiring resistance to treatment, tumor heterogeneity, and restricted delivery of treatments to the central nervous system as a consequence of both the blood-brain barrier and the high interstitial peritumoral pressures $[5,6]$. The high recurrence rate found in patients with GBM is a major clinical challenge. Previous clinical trials have evaluated combination therapy for the treatment of recurrent GBM, and these are better than monotherapies [7-15]. Despite some minor improvements in progressionfree survival (PFS) of recurrent GBM by the introduction of temozolomide (TMZ) and other targeted treatments, no satisfactory improvement in overall survival has been achieved [16]. In addition to a high rate of recurrence, the appearance of new genetic mutations and malignant phenotypes in the process of recurrence increases the difficulty of treatment for recurrent GBM [17, 18]. A comprehensive portrait of the genetic alterations, age distribution, tumor localization and other clinical features in primary and recurrent GBM is needed to better classify tumor profiles. This could lead to an understanding of the characteristics of recurrent GBM, and suggest potential targets for personalized therapeutic strategies.

In the present study, we investigated the characteristics of primary and recurrent GBM, with the goal of developing targeted and individualized treatment for GBM. We used whole transcriptome sequencing to assess 110 samples, including 88 primary GBM and 22 recurrent GBM, with defined molecular subtypes (proneural, neural, classical and mesenchymal). We analyzed the distribution of genetic events and examined the clinical features between the subtypes. We 
found that primary and recurrent GBM showed differences in mutation point distribution and clinical features. Gene set enrichment analysis (GSEA) indicated that the two types of GBM were enriched in different gene sets. Our findings provide a comprehensive portrait of gene alterations, clinical features and gene set enrichment in primary and recurrent GBM, which could be helpful in determining the direction of potential targeted drug therapy.

\section{RESULTS}

\section{Distribution of molecular subtypes in primary and recurrent $\mathrm{GBM}$}

As shown in Figure 1, 36\% of 88 primary GBM belonged to the classical subtype. This was higher than the proportion of this subtype in recurrent GBM (22\%). However, the proportion of the proneural subtype in primary GBM (15\%) was lower than in recurrent GBM (23\%). Samples identified as being of the neural subtype made up $8 \%$ of primary GBM and 9\% of recurrent GBM. Approximately half of primary and recurrent GBM were identified as the mesenchymal subtype ( $41 \%$ and $45 \%$, respectively).

\section{Investigation of gene alterations in primary and recurrent GBM}

IDH1 mutations were found in 12 out of 88 primary GBM (14\%) and 6 of 22 recurrent GBM (27\%). The frequency of ATRX mutations in primary GBM was $10 \%$, and
$18 \%$ in recurrent GBM. TP53 mutation exhibited a slightly higher frequence in $(59 \%)$ recurrent GBM than that of primary GBM (50\%). EGFR mutations were detected in 26\% of primary GBM, and in 18\% of recurrent GBM. (Table 1)

Among proneural subtypes, both primary and recurrent GBM showed a high frequency of IDH1 mutations. TP53 mutations were detected in 5 of 13 proneural primary GBM but in only 1 out of 5 proneural recurrent GBM. Such mutations were also present in 14 of $32(44 \%)$ classical primary GBM, but were even more prevalent in classical recurrent GBM $(80 \%)$.

In the mesenchymal subtype, TP53, EGFR and ATRX mutations were detected in 20 (56\%), $10(28 \%)$ and $3(8 \%)$ out of 36 mesenchymal primary GBM respectively, and 70\%, $10 \%$ and $30 \%$ of 10 mesenchymal recurrent glioblastomas.

\section{Clinical feature of primary and recurrent GBM}

GBM predominantly affected males; the male to female ratio was 1.67 in primary GBM and 1.75 in recurrent GBM (Figure 2A and Table 2). With respect to anatomic localization, the frontal and temporal lobes were the most common sites of primary GBM, accounting for $33 \%$ and $34 \%$, respectively (Figure $2 \mathrm{~B}$ ). However, only $9 \%$ of recurrent GBM were located in the frontal lobe, which was significantly lower than that of recurrent GBM $(P<0.05)$. The most common sites of recurrent GBM were the temporal lobe $(41 \%)$ and other lobes (excluding the frontal and temporal lobes, $41 \%$, Figure 2C). Among the 88 patients with primary GBM, 39 patients were younger

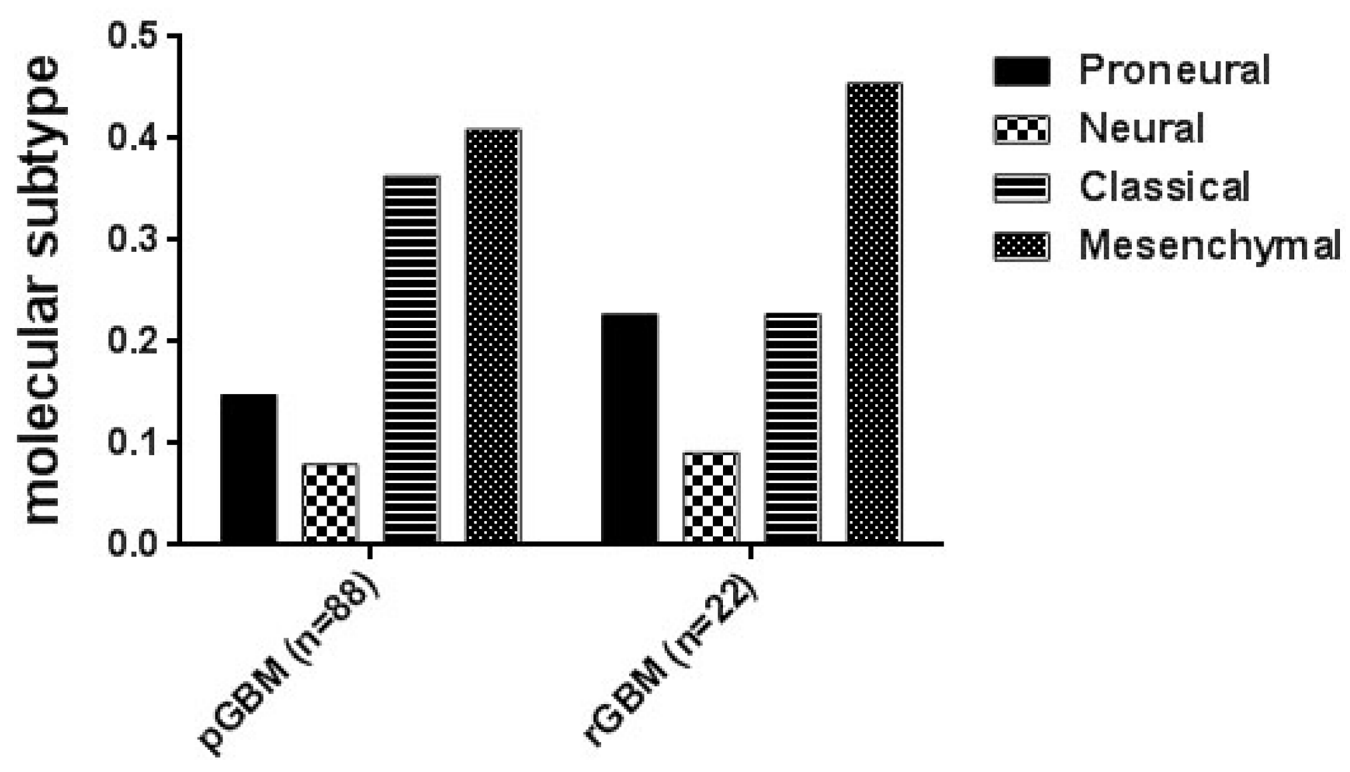

Figure 1: Distribution of molecular subtypes (Proneural, Neural, Classical and Mesenchymal) in primary and recurrent GBM. Molecular subtypes are indicated in different bars. 
Table 1: Gene alterations in primary and recurrent GBM according to their molecular subtypes

\begin{tabular}{|c|c|c|c|c|c|c|c|c|}
\hline & \multicolumn{4}{|c|}{ Primary glioblastoma } & \multicolumn{4}{|c|}{ Recurrent glioblastoma } \\
\hline & Proneural & Neural & Classical & Mesenchymal & Proneural & Neural & Classical & Mesenchymal \\
\hline No. of patients & 13 & 7 & 32 & 36 & 5 & 2 & 5 & 10 \\
\hline IDH 1 mutation & 10 & 0 & 2 & 0 & 4 & 0 & 1 & 1 \\
\hline TP53 mutation & 5 & 5 & 14 & 20 & 1 & 1 & 4 & 7 \\
\hline EGFR mutation & 4 & 1 & 8 & 10 & 2 & 0 & 1 & 1 \\
\hline ATRX mutation & 2 & 2 & 2 & 3 & 0 & 0 & 1 & 3 \\
\hline
\end{tabular}

\section{A}
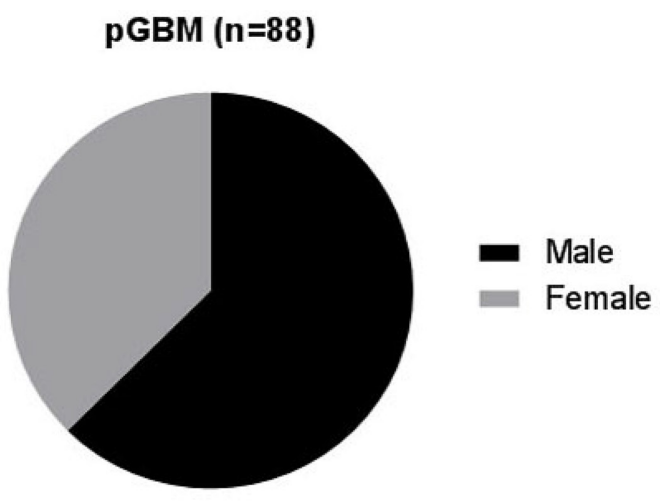

B
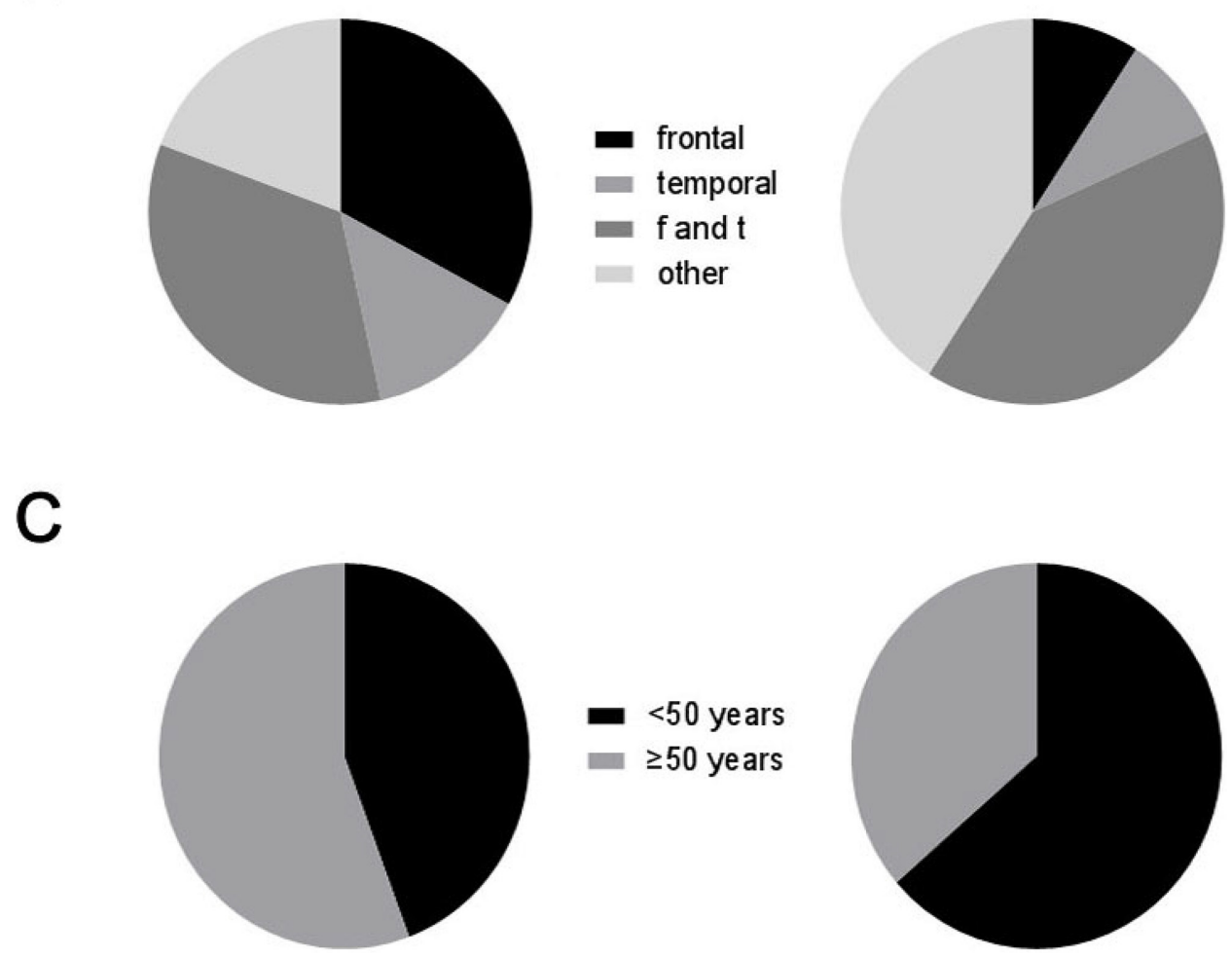

Figure 2: Clinical features of primary and recurrent GBM. A. Distribution of gender with molecular subtypes of GBM; B. Distribution of anatomic localization with molecular subtypes of GBM (f and t: co-involving the frontal and temporal lobes. other lobes: excluding the frontal and temporal lobes); C. Age cohort of patients with four molecular subtypes of GBM. 
Table 2: Clinical features of patients with primary and recurrent GBM

\begin{tabular}{|c|c|c|c|c|c|c|c|c|}
\hline \multirow[b]{2}{*}{ No. of patients } & \multicolumn{2}{|c|}{ Age (years) } & \multicolumn{2}{|c|}{ Gender } & \multicolumn{4}{|c|}{ anatomical location } \\
\hline & $<\mathbf{5 0}$ & $\geq \mathbf{5 0}$ & Male & Female & $\begin{array}{c}\text { Frontal } \\
\text { lobe }\end{array}$ & $\begin{array}{c}\text { Temporal } \\
\text { lobe }\end{array}$ & $\begin{array}{c}\text { Both } \\
\text { lobes* }\end{array}$ & Other lobes \\
\hline Primary GBM & 39 & 49 & 55 & 33 & 29 & 30 & 12 & 17 \\
\hline percentage & $44 \%$ & $56 \%$ & $63 \%$ & $37 \%$ & $33 \%$ & $34 \%$ & $14 \%$ & $19 \%$ \\
\hline Recurrent GBM & 14 & 8 & 14 & 8 & 2 & 9 & 2 & 9 \\
\hline percentage & $64 \%$ & $36 \%$ & $64 \%$ & $36 \%$ & $9 \%$ & $41 \%$ & $9 \%$ & $41 \%$ \\
\hline
\end{tabular}

*Both lobes: co-involved frontal and temporal lobes.

than 50 years of age, and 49 patients were 50 years of age or older (Figure 2B). Among the 22 patients with recurrent GBM, 14 patients were younger than 50 years and 8 patients were 50 years of age or older (Figure 2C). The mean age of patients diagnosed with primary GBM was $49.61 \pm 1.346$ years, and with recurrent GBM, the mean age was $47.73 \pm 1.782$ years.

\section{Gene set enrichment analysis of primary and recurrent GBM}

We performed GSEA on the whole transcriptome sequencing of 88 primary GBM and 22 recurrent GBM. Our results indicate that gene sets related to TRANSLATION $(P<0.001)$, RNA PROCESSING $(P=0.024)$ and CELLULAR_BIOSYNTHETIC_PROCESS $(P=0.016)$ were significantly enriched in the primary GBM, while DNA DAMAGE RESPONSESIGNAL TRANSDUCTION $(P<$ 0.001), DNA DAMAGE_CHECKPOINT $(P=0.010)$ and CHROMOSOME_SEGREGATION $(P=0.013)$ gene sets were enriched in recurrent GBM (Figure 3 and Supplementary Table S1).

\section{DISCUSSION}

Glioblastoma multiforme (GBM) is the most common and aggressive malignant primary brain tumor in adults. Despite treatment involving chemotherapy, radiation and surgery, GBM exhibits a median survival rate of 15 months $[3,4,19]$. An important factor in the poor prognosis of primary GBM is a high recurrence rate [20]. Currently, treatments for recurrent GBM mainly involve repeated resection, further chemotherapy and focal irradiation [21, $22]$. With the accumulation of knowledge regarding the molecular and genetic profile of GBM, some molecularly targeted therapies, such as growth factor inhibitors or TMZ, have led to some progress in treatment $[3,16]$. However, despite these advances in treatment strategies, the majority of patients suffered recurrent GBM, with a subsequent median survival of approximately 6 months [23-26]. Therefore, insight into the differences between primary and recurrent glioblastoma from a molecular and clinical perspective, and exploration of more effective molecular targets for therapy is urgently. To date, the distinction between primary and recurrent GBM has not been systematically investigated. In the present study, 88 primary and 22 recurrent GBM were investigated using whole transcriptome sequencing combined with analysis of clinical data. When the 110 tumors were divided into molecular subtypes, we found that the proportion of proneural subtype was more frequent in recurrent GBM than that of primary GBM, while the classical subtype showed an opposite result. The incidence of neural and mesenchymal subtypes between primary and recurrent GBM remained similar. These results suggest that the proneural subtype was more likely to recurrent when compared to the other molecular subtypes. The classical subtype exhibited the opposite pattern of incidence.

The frequency of IDH1 mutations in recurrent GBM was nearly twice that of primary GBM, and distribution of this group of mutations was particularly high in the proneural subtype. TP53 mutations were more likely to be found in classical and mesenchymal recurrent GBM than in primary GBM. However, such mutations were rare in proneural recurrent GBM. The phenomena of variable distribution of molecular subtypes and gene signatures between primary and recurrent GBM suggests that these genetic events might play important roles in the recurrent progression of primary GBM. For example, classical and mesenchymal primary GBM exhibiting TP53 mutation had a high risk of recurrence, whereas proneural primary GBM carrying TP53 mutation were less likely to relapse.

The ratio of male to female incidence of primary and recurrent GBM was approximately the same, demonstrating that there was no difference in recurrence of GBM depending on gender. With respect to anatomic localization, $33 \%$ of primary GBM were located in frontal lobe, whereas only $9 \%$ of recurrent GBM were found there. Conversely, other lobes, especially temporal lobe, were more common sites for recurrent GBM than primary GBM. These findings suggest that tumors in frontal lobe had a low recurrent rate. There was no significant difference in mean age between primary and recurrent GBM. However, when we used 50-year-old as a boundary line to divided into 2 groups, less than 50 and more than or equal to 50 , we found that $64 \%$ of patients with recurrent 
A Primary GBM $(n=88)$
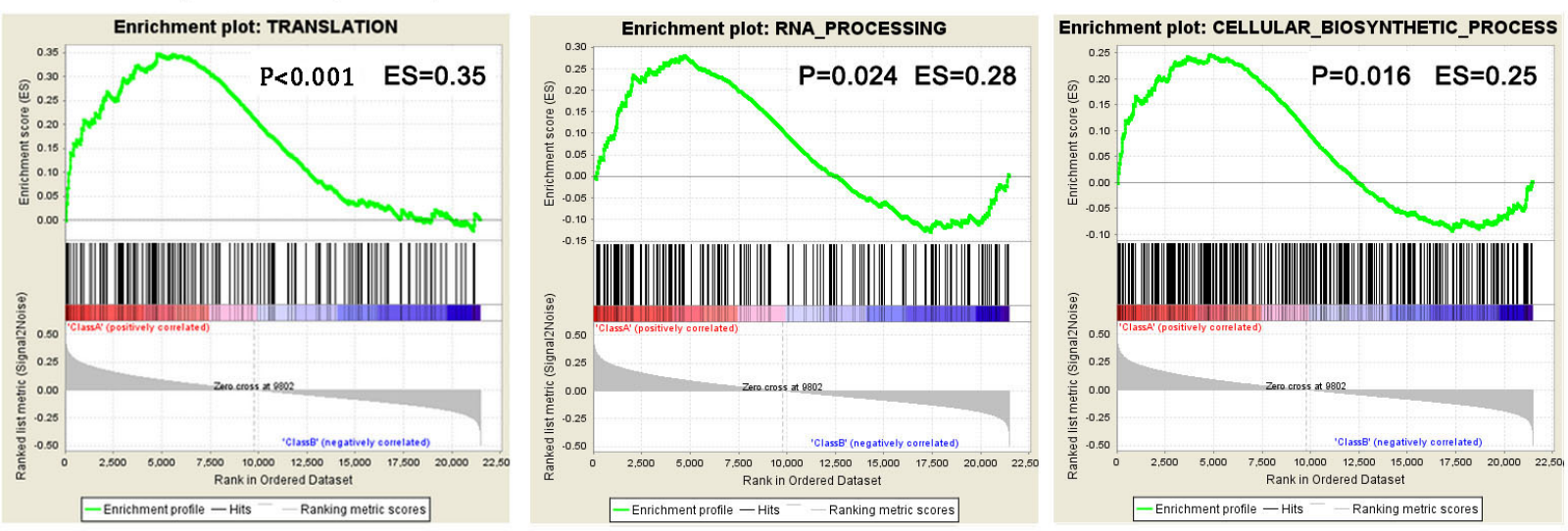

\section{B Recurrent GBM ( $\mathrm{n}=22)$}
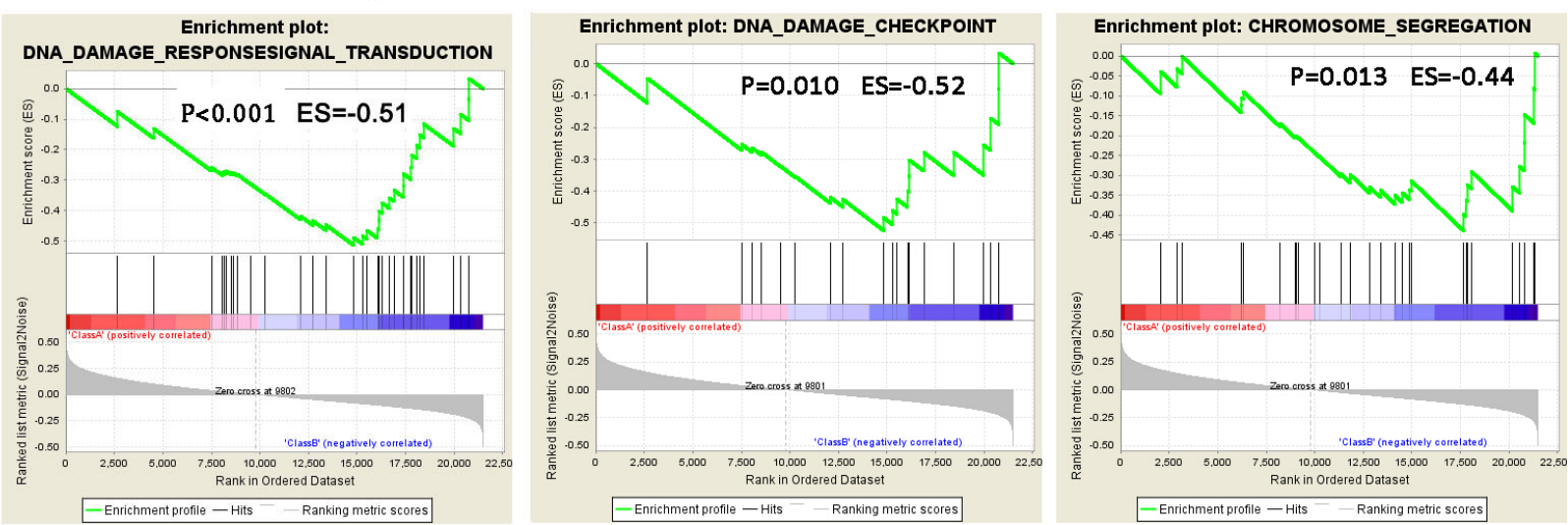

Figure 3: Presence of representative gene sets related to biological processes analyzed by GSEA. A. Gene sets related to biological processes in $88 \mathrm{pGBM}$; B. Gene sets related to biological processes in $22 \mathrm{rGBM}$.

GBM were younger than 50 years old, compared with $44 \%$ of primary GBM. Because there is no difference in age between primary and recurrent GBM in the whole cohort, we cannot conclude that people younger than 50 years old have a higher tendency towards recurrence. However, the results above suggest that the recurrence rate of primary GBM does not increase with age.

Aimed for finding the enriched gene sets among the differentially expressed genes between primary and recurrent tumors, GSEA was performed for primary and recurrent GBM in this study. Enriched gene sets related to TRANSLATION, RNA_PROCESSING and CELLULAR BIOSYNTHETIC_PROCESS were found in primary GBM. These are related to protection and progression of primary GBM, while DNA DAMAGE RESPONSESIGNAL TRANSDUCTION, DNA_DAMAGE_CHECKPOINT and CHROMOSOME_SEGREGATION gene sets relating to recurrence of tumor cells were enriched in recurrent GBM. Compared to primary GBM, recurrent tumors displayed significant biological progression, including DNA damage repair, cell metabolic process and other rebuilt procession of recurrent tumor cells. This was consistent with the clinical progression of recurrent GBM relapsed from the residual cancer cells after treatments.

Our study comprehensively characterizes the distinction in molecular subtypes, gene signatures and clinical features between primary and recurrent GBM. Although past efforts have not significantly improved the prognosis of recurrent GBM, increasing insight into primary and recurrent GBM can help us to enhance timely intervention and reduce the recurrence rate of GBM, as well as improving molecularly targeted therapies.

\section{MATERIALS AND METHODS}

\section{Tumor samples}

A total of 110 glioblastoma multiforme (GBM) samples from the Chinese Glioma Genome Atlas were included in this study, consisting of 88 primary GBM (pGBM) and 22 recurrent GBM (rGBM). Tumor tissue samples were obtained by surgical resection. All pGBM and rGBM cases were classified by two neuropathologists according to the 2007 WHO 
classification guidelines and Scherer [27]. Only samples with greater than $80 \%$ tumor cells were selected. All patients provided written informed consent, and the study was approved by the ethics committees of the participating hospitals.

\section{Whole transcriptome sequencing}

Whole transcriptome sequencing was performed as described previously [28]. Briefly, total RNA was isolated from homogenized frozen tissue samples using the RNeasy Mini Kit (Qiagen) according to the manufacturer's instructions. RNA purity was checked using a 2100 Bioanalyzer (Agilent Technologies) and only high quality samples with an RNA Integrity Number (RIN) value greater than or equal to 7.0 were used to construct the sequencing library. The subsequent sequencing steps included end repair, adapter ligation, size selection and polymerase chain reaction enrichment. The length of DNA fragment was measured using a 2100 Bioanalyzer, with a median insert size of 200 nucleotides. The libraries were sequenced on the Illumina HiSeq 2000 platform using the 101-bp pair-end sequencing strategy. Short sequence reads were aligned to the human reference genome $(\mathrm{Hg}$ 19 Refseq) using the Burrows-Wheeler Aligner (BWA, Version 0.6.2-r126). SnpEff software was used to annotate genetic variance [29].

\section{Gene set enrichment analysis}

To determine the gene sets related to particular biological processes present in pGBM and rGBM, gene expression profiling and gene set enrichment analysis (GSEA) was performed as described previously [30].

\section{Statistical analysis}

Student's $t$-test was performed using SPSS 13.0. All data are presented as the mean \pm SEM. A $P$-value of $<0.05$ was considered significant.

\section{ACKNOWLEDGMENTS}

This work was supported by grants from the National Natural Science Foundation of China (No.81402056), National Natural Science Foundation of China(No.81402068), National Natural Science Foundation of China (No.81302184), National High Technology Research and Development Program of China (863) (No.2012AA02A508).

\section{CONFLICTS OF INTEREST}

All authors report no conflicts of interest relevant to this article.

\section{REFERENCES}

1. Ohgaki H, Dessen P, Jourde B, Horstmann S, Nishikawa T, Di Patre PL, Burkhard C, Schuler D, Probst-Hensch NM, Maiorka PC, Baeza N, Pisani P, Yonekawa Y, et al. Genetic pathways to glioblastoma: a population-based study. Cancer research. 2004; 64:6892-6899.

2. Ohgaki H, Kleihues P. Epidemiology and etiology of gliomas. Acta neuropathologica. 2005; 109:93-108.

3. Stupp R, Dietrich PY, Ostermann Kraljevic S, Pica A, Maillard I, Maeder P, Meuli R, Janzer R, Pizzolato G, Miralbell R, Porchet F, Regli L, de Tribolet N, et al. Promising survival for patients with newly diagnosed glioblastoma multiforme treated with concomitant radiation plus temozolomide followed by adjuvant temozolomide. Journal of clinical oncology : official journal of the American Society of Clinical Oncology. 2002; 20:1375-1382.

4. Lacroix M, Abi-Said D, Fourney DR, Gokaslan ZL, Shi W, DeMonte F, Lang FF, McCutcheon IE, Hassenbusch SJ, Holland E, Hess K, Michael C, Miller D, Sawaya R. A multivariate analysis of 416 patients with glioblastoma multiforme: prognosis, extent of resection, and survival. Journal of neurosurgery. 2001; 95:190-198.

5. Reardon DA, Rich JN, Friedman HS, Bigner DD. Recent advances in the treatment of malignant astrocytoma. Journal of clinical oncology : official journal of the American Society of Clinical Oncology. 2006; 24:1253-1265.

6. Wen PY, Kesari S. Malignant gliomas in adults. The New England journal of medicine. 2008; 359:492-507.

7. Brandes AA, Ermani M, Turazzi S, Scelzi E, Berti F, Amista P, Rotilio A, Licata C, Fiorentino MV. Procarbazine and high-dose tamoxifen as a second-line regimen in recurrent high-grade gliomas: a phase II study. Journal of clinical oncology : official journal of the American Society of Clinical Oncology. 1999; 17:645-650.

8. Vredenburgh JJ, Desjardins A, Herndon JE 2nd, Marcello J, Reardon DA, Quinn JA, Rich JN, Sathornsumetee S, Gururangan S, Sampson J, Wagner M, Bailey L, Bigner DD, et al. Bevacizumab plus irinotecan in recurrent glioblastoma multiforme. Journal of clinical oncology : official journal of the American Society of Clinical Oncology. 2007; 25:4722-4729.

9. Doherty L, Gigas DC, Kesari S, Drappatz J, Kim R, Zimmerman J, Ostrowsky L, Wen PY. Pilot study of the combination of EGFR and mTOR inhibitors in recurrent malignant gliomas. Neurology. 2006; 67:156-158.

10. van den Bent MJ, Taal W. Are we done with dose-intense temozolomide in recurrent glioblastoma? Neuro-oncology. 2014; 16:1161-1163.

11. Dresemann G. Imatinib and hydroxyurea in pretreated progressive glioblastoma multiforme: a patient series. Annals of oncology : official journal of the European Society for Medical Oncology / ESMO. 2005; 16:1702-1708. 
12. Reardon DA, Quinn JA, Vredenburgh JJ, Gururangan S, Friedman AH, Desjardins A, Sathornsumetee S, Herndon JE 2nd, Dowell JM, McLendon RE, Provenzale JM, Sampson JH, Smith RP, et al. Phase 1 trial of gefitinib plus sirolimus in adults with recurrent malignant glioma. Clinical cancer research : an official journal of the American Association for Cancer Research. 2006; 12:860-868.

13. Friedman HS, Prados MD, Wen PY, Mikkelsen T, Schiff D, Abrey LE, Yung WK, Paleologos N, Nicholas MK, Jensen R, Vredenburgh J, Huang J, Zheng M, Cloughesy T. Bevacizumab alone and in combination with irinotecan in recurrent glioblastoma. Journal of clinical oncology : official journal of the American Society of Clinical Oncology. 2009; 27:4733-4740.

14. Reardon DA, Egorin MJ, Quinn JA, Rich JN, Gururangan S, Vredenburgh JJ, Desjardins A, Sathornsumetee S, Provenzale JM, Herndon JE 2nd, Dowell JM, Badruddoja MA, McLendon RE, et al. Phase II study of imatinib mesylate plus hydroxyurea in adults with recurrent glioblastoma multiforme. Journal of clinical oncology : official journal of the American Society of Clinical Oncology. 2005; 23:9359-9368.

15. Reardon DA, Dresemann G, Taillibert S, Campone M, van den Bent M, Clement P, Blomquist E, Gordower L, Schultz H, Raizer J, Hau P, Easaw J, Gil M, et al. Multicentre phase II studies evaluating imatinib plus hydroxyurea in patients with progressive glioblastoma. British journal of cancer. 2009; 101:1995-2004.

16. Johnson DR, O'Neill BP. Glioblastoma survival in the United States before and during the temozolomide era. Journal of neuro-oncology. 2012; 107:359-364.

17. Cloughesy TF, Cavenee WK, Mischel PS. Glioblastoma: from molecular pathology to targeted treatment. Annual review of pathology. 2014; 9:1-25.

18. Ferrara N, Hillan KJ, Novotny W. Bevacizumab (Avastin), a humanized anti-VEGF monoclonal antibody for cancer therapy. Biochemical and biophysical research communications. 2005; 333:328-335.

19. Buckner JC. Factors influencing survival in high-grade gliomas. Seminars in oncology. 2003; 30:10-14.

20. Weller M, Cloughesy T, Perry JR, Wick W. Standards of care for treatment of recurrent glioblastoma - are we there yet? Neuro-oncology. 2013; 15:4-27.

21. Kast RE, Boockvar JA, Bruning A, Cappello F, Chang WW, Cvek B, Dou QP, Duenas-Gonzalez A, EfferthT, Focosi D, Ghaffari SH, Karpel-Massler G, Ketola K, et al. A conceptually new treatment approach for relapsed glioblastoma: coordinated undermining of survival paths with nine repurposed drugs (CUSP9) by the International Initiative for Accelerated Improvement of Glioblastoma Care. Oncotarget. 2013; 4:502-530.

22. Nonnenmacher L, Westhoff MA, Fulda S, KarpelMassler G, Halatsch ME, Engelke J, Simmet T, Corbacioglu S, Debatin KM. RIST: a potent new combination therapy for glioblastoma. International journal of cancer Journal international du cancer. 2015; 136:E173-187.

23. Wong ET, Hess KR, Gleason MJ, Jaeckle KA, Kyritsis AP, Prados MD, Levin VA, Yung WK. Outcomes and prognostic factors in recurrent glioma patients enrolled onto phase II clinical trials. Journal of clinical oncology : official journal of the American Society of Clinical Oncology. 1999; 17:2572-2578.

24. Lamborn KR, Yung WK, Chang SM, Wen PY, Cloughesy TF, DeAngelis LM, Robins HI, Lieberman FS, Fine HA, Fink KL, Junck L, Abrey L, Gilbert MR, et al. Progression-free survival: an important end point in evaluating therapy for recurrent high-grade gliomas. Neurooncology. 2008; 10:162-170.

25. Ballman KV, Buckner JC, Brown PD, Giannini C, Flynn PJ, LaPlant BR, Jaeckle KA. The relationship between six-month progression-free survival and 12-month overall survival end points for phase II trials in patients with glioblastoma multiforme. Neuro-oncology. 2007; 9:29-38.

26. Balmaceda C, Peereboom D, Pannullo S, Cheung YK, Fisher PG, Alavi J, Sisti M, Chen J, Fine RL. Multiinstitutional phase II study of temozolomide administered twice daily in the treatment of recurrent high-grade gliomas. Cancer. 2008; 112:1139-1146.

27. Scherer HJ. Cerebral astrocytomas and their derivatives. Am. J Cancer. 1940; 40:159-198.

28. Bao ZS, Chen HM, Yang MY, Zhang CB, Yu K, Ye WL, Hu BQ, Yan W, Zhang W, Akers J, Ramakrishnan V, Li J, Carter B, et al. RNA-seq of 272 gliomas revealed a novel, recurrent PTPRZ1-MET fusion transcript in secondary glioblastomas. Genome research. 2014; 24:1765-1773.

29. Li H, Durbin R. Fast and accurate short read alignment with Burrows-Wheeler transform. Bioinformatics. 2009; 25:1754-1760.

30. Subramanian A, Tamayo P, Mootha VK, Mukherjee S, Ebert BL, Gillette MA, Paulovich A, Pomeroy SL, Golub TR, Lander ES, Mesirov JP. Gene set enrichment analysis: a knowledge-based approach for interpreting genome-wide expression profiles. Proceedings of the National Academy of Sciences of the United States of America. 2005; 102:15545-15550. 